\title{
HPLC/PDA-ESI/MS Identification of Phenolic Acids, Flavonol Glycosides and Antioxidant Potential in Blueberry, Blackberry, Raspberries and Cranberries
}

\author{
ZORIȚA DIACONEASA ${ }^{1, *}$, RANGA FLORICA ${ }^{1}$, DUMITRIȚA RUGINĂ ${ }^{2}$, CUIBUS LUCIAN ${ }^{1}$, \\ CARMEN SOCACIU, ${ }^{1, *}$ \\ ${ }^{1}$ Faculty of Food Science and Technology, University of Agricultural Science and Veterinary Medicine Cluj-Napoca, Romania, Calea \\ Mănăştur 3-5, Cluj-Napoca, Romania \\ ${ }^{2}$ Faculty of Veterinary Medicine, University of Agricultural Science and Veterinary Medicine Cluj-Napoca, Romania, Calea Mănăştur \\ 3-5, Cluj-Napoca, Romania \\ *Corresponding author: zorita.sconta@usamvcluj.ro; carmen.socaciu@usamvcluj.ro
}

Received October 04, 2014; Revised October 17, 2014; Accepted October 22, 2014

\begin{abstract}
Berry fruits are known to be a rich source of natural compounds which provide them many health benefits. The most common compounds that occur in berries are vitamins, flavonoids, anthocyanins and phenolic acids. The objective of this work was to identify and compare the phenolic acids, flavonol glycosides content and antioxidant potential in blueberry, blackberry, raspberries and cranberries. Berries methanolic extracts were analyzed by HPLC/PDA-ESI/MS. Antioxidant activity was determinate using FRAP assay. Antioxidant activity of analyzed berries varied considerably. Blueberry extract demonstrated the highest ferric reducing antioxidant potential (48.3 $\mu \mathrm{M} \mathrm{Fe} / \mathrm{g})$ while lower values were obtained for cranberries extract $\left(19.6 \mu \mathrm{M} \mathrm{Fe}^{2} / \mathrm{g}\right)$. Berries extracts were characterized by the presence of 16 compounds in different ratios. Blueberry extract was characterized by the presence of a large amount of phenolic acids such as chlorogenic and caffeic acid while raspberries extract was found to have high amount of ellagic acid. All analyzed berries contain higher levels of bioactive compounds which are responsible for their antioxidant potential. Based on quantitative analysis of phenolics these fruits can be highly recommended for daily consumption.
\end{abstract}

Keywords: berries, phenolic acids, flavonol glycosides, antioxidant activity, HPLC-ESI/MS

Cite This Article: ZORIȚA DIACONEASA, RANGA FLORICA, DUMITRIȚA RUGINĂ, CUIBUS LUCIAN, and CARMEN SOCACIU, "HPLC/PDA-ESI/MS Identification of Phenolic Acids, Flavonol Glycosides and Antioxidant Potential in Blueberry, Blackberry, Raspberries and Cranberries.” Journal of Food and Nutrition Research vol. 2, no. 11 (2014): 781-785. doi: 10.12691/jfnr-2-11-4.

\section{Introduction}

Daily consumption of fruits and vegetables is consistently associated with reductions in the incidence of degenerative diseases, by having antioxidant, antiinflammatory and anticarcinogenic activity [1-10]. Lock, K et al., 2005 [11], reported that daily consumption of 600 $\mathrm{g}$ of fruits or vegetables can prevent the risk of coronary heart disease by more than 31\% and ischemic stroke by $19 \%$.

Berries and vegetables contain an important amount of fiber and high concentrations of phenolic acids, flavonoids, vitamins, or minerals. Phenolic acids and flavonoids are bioactive compounds that contribute to all those biological proprieties and exhibit health benefits. The phenolic acids that provide these beneficial effects are: chlorogenic acid, gallic acid, coumaric acid, caffeic acid [12]. Flavonoids are polyphenolic compounds, part of a large group of secondary plant metabolites. The most common flavonoids found in berries and vegetables are the tannins, phenolic acids and flavonols. The most abundant are anthocyanins which also give fruits their nice and attractive colors. All those compounds were found to be presents in all sources in an acylated (with aliphatic or aromatic acids) or glycosylated form. Due to their complexity, the characterization of polyphenols, their identification and also antioxidant potential is a challenging task for many researchers because all these parameters/methods/analysis are influenced by the type of sugar moiety, degree of glycosylation as well as acylation of analyzed compound [13,14,15]. Berries, including blueberry, blackberry, raspberries and cranberries, are known for their high antioxidant potential being a rich source of dietary antioxidants[16,17]. Their antioxidant activity is associated to some extent with the number of hydroxyl groups in their molecular structure (Figure 1). In a recent publish Zheng and Wang [18] have evaluated and compare the antioxidant activity of phenolic acid found in different berries. They result showed that chlorogenic acid have lower antioxidant activity compared to that of caffeic acid, also vanillic acid had values similar to the values obtained for p-coumaric acid. Those results can be 
influenced by the substitution of the 3-hydroxyl group by a methoxy group activity [19]. For their identification or quantification different techniques have been used, such as reversed-phase high-performance liquid chromatography and MALDI-TOF MS coupled with mass spectrometric (MS/MS) analysis [20,21], high performance thin layer chromatography (HPTLC) [22] and also NMR [23]. The selected berries analyzed in this study are the most popular berries consumed in Romania. The aim of our study was to identify and compares the amount of phenolic acids and flavonoid glycosides from Romanian berries harvested from spontaneous flora with available published data. To our knowledge this is the first time that identification of phenolics and flavonoids in spontaneous berry from Romania (blueberry, blackberry, raspberries, and cranberries) is reported as the subject of a scientific paper.

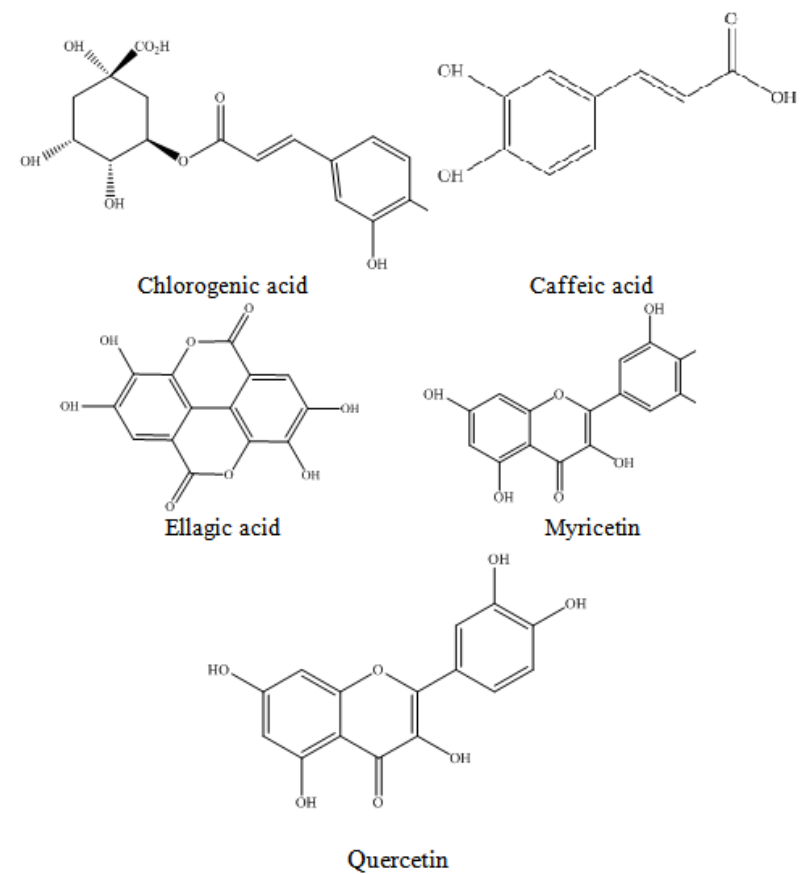

Figure 1. Structure of phenolics in, blueberry, raspberry, blackberry and cranberry

\section{Materials and Methods}

\subsection{Materials}

Reagents. Acetonitrile and formic acid used for HPLCMS analysis were purchased from Sigma-Aldrich (Darmstadt, Germany). All solvents used for extraction were of analytical grade. All other chemicals were purchased from Merck (Darmstadt, Germany).

Plant material. Blueberries (Vaccinum corymbosum), raspberries (Rubus idaeus), cranberries (Vaccinum vitisidaea), and blackberries (Rubus fruticosus) were purchased from local farmers in the west of Transylvania. The samples were stored in a freezer at $-20^{\circ} \mathrm{C}$ until they were analyzed.

Extraction methods. For the extraction, $1 \mathrm{~g}$ of each berries, were grinded and mixed with 10 methanol/formic acid (99:1, v/v). The pellets were centrifuged at $3500 \mathrm{rpm}$ for $10 \mathrm{~min}$ and re-extracted until the extraction solvents became colorless. All supernatants were combined and concentrated at $35^{\circ} \mathrm{C}$ under reduced pressure (Rotavapor ${ }^{\circledR}$ model R-124; Buchi, Flawil, Switzerland). The evaporated samples were solubilized in a known amount of acidified methanol, filtered through $0.45 \mu \mathrm{m}$ Millipore nylon filter and used for HPLC analysis.

\subsection{Methods}

Ferric reducing antioxidant power. Antioxidant potential of analyzed berries was performed according to Benzie and Strain, 1999 [24] with minor modifications. Berries antioxidants can reduce the damaging effects of hydroxyl radicals by removing their free electrons to form $\mathrm{H}_{2} \mathrm{O}$ and the antioxidant radicals which are less aggressive. This assay depends on the reduction of ferric tripyridyltriazine $\left(\mathrm{Fe}^{3+}-\mathrm{TPTZ}\right)$ complex to ferrous tripyridyltriazine $\left(\mathrm{Fe}^{2+}-\mathrm{TPTZ}\right)$. The reduction of ferric tripyridyltriazine $\left(\mathrm{Fe}^{3+}-\mathrm{TPTZ}\right)$ complex to ferrous tripyridyltriazine $\left(\mathrm{Fe}^{2+}-\mathrm{TPTZ}\right)$ complex occurs in acidic conditions ( $\mathrm{pH}$ 3.6) to maintain iron solubility. Briefly, FRAP reagent consisted of $2.5 \mathrm{ml}$ of TPTZ (10 mM in 40 $\mathrm{mM} \mathrm{HCl}$ ) solution, $25 \mathrm{ml}$ sodium acetate buffer (300 mM, $\mathrm{pH}$ 3.6), and $2.5 \mathrm{ml} \mathrm{FeCl}_{3}$ solution (20 mM). The analysis was carried out 96 -well clear plates. FRAP reagent $(180 \mu \mathrm{l})$ was mixed with the sample or blank $(20 \mu \mathrm{l})$ and added to the plate. The plates ere incubated for about 3 minutes at $37^{\circ} \mathrm{C}$ and the absorbance of ferrous tripyridyltriazine complex was read at $593 \mathrm{~nm}$ with a microplate reader. Antioxidant potential corresponding to the capacity of the sample to reduce iron was expressed as $\mu \mathrm{M} \mathrm{Fe}^{2}$ equivalent per gram of berry sample ( $\mu \mathrm{M} \mathrm{Fe}{ }^{2}$ equivalent /g FW).

HPLC-PDA/-ESI-MS identification and quantification of phenolic acids and flavonol glycosides. HPLC analysis was performed on a Agilent 1200 system equipped with a binary pump delivery system LC-20 AT (Prominence), a degasser DGU-20 A3 (Prominence), diode array SPD-M20 A UV-VIS detector (DAD) and a Eclipse XDB C18 column ( $4 \mu \mathrm{m}, 4.6$ x $150 \mathrm{~mm}$ ) was used. The mobile phase consisted in: solvent A - bidistilled water and $0.1 \%$ acetic acid/acetonitrile (99/1) v/v, B acetonitrile and acetic acid $0.1 \%$. The gradient elution system was: 0-2 min, isocratic with 5\% (v/v) eluent B; 218 min, linear gradient from $5 \%$ to $40 \%(\mathrm{v} / \mathrm{v})$ eluent $\mathrm{B}$; 18-20 min, linear gradient from $40 \%$ to $90 \%(\mathrm{v} / \mathrm{v})$ eluent B; 20-24 min, isocratic on 90\% (v/v) eluent B; 24-25 min, linear gradient from $90 \%$ to $5 \%(\mathrm{v} / \mathrm{v}$ ) eluent B; $25-30 \mathrm{~min}$, isocratic on $5 \%(\mathrm{v} / \mathrm{v})$ eluent B. Flow rate was $0.5 \mathrm{ml} / \mathrm{min}$ and column temperature was maintained at $25^{\circ} \mathrm{C}$. The chromatograms were monitored at 280 respectively 340 $\mathrm{nm}$. The compounds identification and peak assignments were done based on their retention times, UV-VIS spectra and also comparing with standards (chlorogenic acid, caffeic acid, quercetin-rutinoside, quercetin-glucoside, ellagic acid, myricetin, all purchased from Sigma-Aldrich, Darmstadt, Germany) and published data. As a confirmation the samples were analyzed by HPLC-ESIMS.

The mass spectrometric data were obtained using a singlequadrupole 6110 mass spectrometer (Agilent Technologies, Chelmsford, MA, USA) equipped with an ESI probe. The measurements were performed in the positive mode with an ion spray voltage of $3000 \mathrm{~V}$, and a capillary temperature of $350^{\circ} \mathrm{C}$. Data were collected in full scan mode within the range 280 to $1000 \mathrm{~m} / \mathrm{z}$. 
Quantification of flavonol glycosides was carried using a rutin standard, and the various compounds were expressed as rutin equivalents. The phenolic acids quantification was done using a chlorogenic acid standard curve.

\section{Results and Discussions}

Total FRAP Antioxidant Activity. The total antioxidant potential values obtained for all berries analyzed was high; the numbers are showed in Table 1. Blueberry and blackberry extract demonstrated the highest ferric reducing antioxidant potential while lower values were obtained for raspberries and cranberries. The obtained results are in agreement with the reported results published by Gina Borges \& al. 2010 [25]. They report FRAP values as $30.0 \mu \mathrm{M} \mathrm{Fe} / \mathrm{g}$ for blueberry, 27.7 and $18.6 \mu \mathrm{M} \mathrm{Fe}^{2} / \mathrm{g}$ for raspberries respectively cranberries. In a previously study Moyer \& al. 2002 [26] has obtained similar FRAP values for blueberries and raspberries. The FRAP values of our study obtained for blueberry extract (48.3 $\mu \mathrm{M} \mathrm{Fe} \mathrm{Fe}^{2+} / \mathrm{g}$ ) was found to be higher than those reported by Koca and Karadeniz [27] (7.41-13.69 $\mu \mathrm{M}$ $\mathrm{Fe}^{2+} / \mathrm{g}$ and lower the values reported by Castrejón \& al., 2008 [28] (34.45-57.92 $\left.\mu \mathrm{M} \mathrm{Fe} \mathrm{Fe}^{2+} / \mathrm{g}\right)$. The differences between published results and the results obtained in this study vary due to numerous factors including extraction method and solvents, berries variety, degree of ripeness and also storage conditions.

Table 1. Antioxidant activity of analyzed berries measured by FRAP assay

\begin{tabular}{|l|l|l|}
\hline Sample no & Berry & FRAP $(\mu \mathrm{m} \mathrm{Fe} 2 / \mathrm{g})$ \\
\hline 1 & Blueberry & $48.3 \pm 0.81$ \\
\hline 2 & Blackberry & $38.7 \pm 0.64$ \\
\hline 3 & Raspberries & $26.3 \pm 0.12$ \\
\hline 4 & Cranberries & $19.6 \pm 0.69$ \\
\hline \multicolumn{2}{|l}{ The data expressed as Mean \pm SD }
\end{tabular}

Identification of phenolic acids, flavonol glycoside by HPLC-ESI-MS. The phenolic acids and flavonol glycosides content and composition from several berry commonly cultivated in Romania were analyzed. The compounds identification was based on their retention times, their UV-Vis absorption spectra, mass spectrometry of analyzed molecules and also available literature data $[17,25,29]$. The identified compounds (16) are showed in Table 2. Quercetin-galactoside, quercetin-rutinoside and myricetin-galactoside were found to be the major flavonol in the analyzed berries samples.

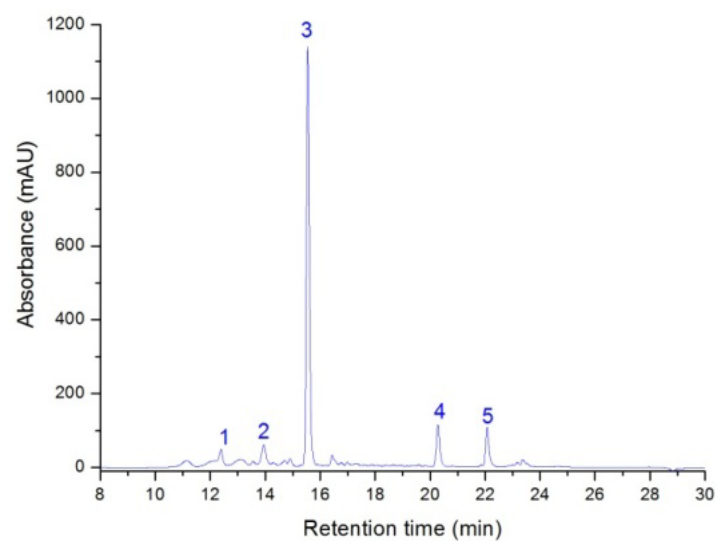

Figure 2. HPLC chromatogram of blueberry extracts
Blueberries. The separation of phenolic acids and flavonol glycosides in blueberries extract is shown in Figure 2. The flavonol glycosides compounds found in blueberries extract were identified as quercetin-rutinoside (rutin) (peak 3), quercetin-galactoside (peak 4), and quercetin-glucoside (peak 5).

The highest flavonol glycosides content in blueberries is represented by quercetin-rutinoside (rutin) 65.96 $\mathrm{mg} / 100 \mathrm{~g} \mathrm{FW}$, value which is higher than ones reported by M.J. Cho \& al, 2004 [17]. In same study, M.J. Cho \& al, 2004 [17] identified the main flavonol glycosides in highbush blueberries as myricetin 3-galactoside/glucoside, myricetin 3-rhamnoside, quercetin 3-galactoside, quercetin 3-glucoside, quercetin 3-rutinoside, and quercetin 3-acetylrhamnoside. Kader \& al, 1996 [30] reported in a previously study the presence in highbush blueberries of 3 main flavonol glycosides as quercetin 3glucoside, quercetin 3-galactoside and quercetin 3rhamnoside, which is in good correlation with our result, except that we identified also quercetin-rutinoside in analyzed blueberries. The analyzed blueberry extract was also characterized by the presence of a large amount of phenolic acids such as chlorogenic (5.13 mg/100 g FW) and caffeic acid (7.5 mg/100 g FW) (early elution peak 1, 2).

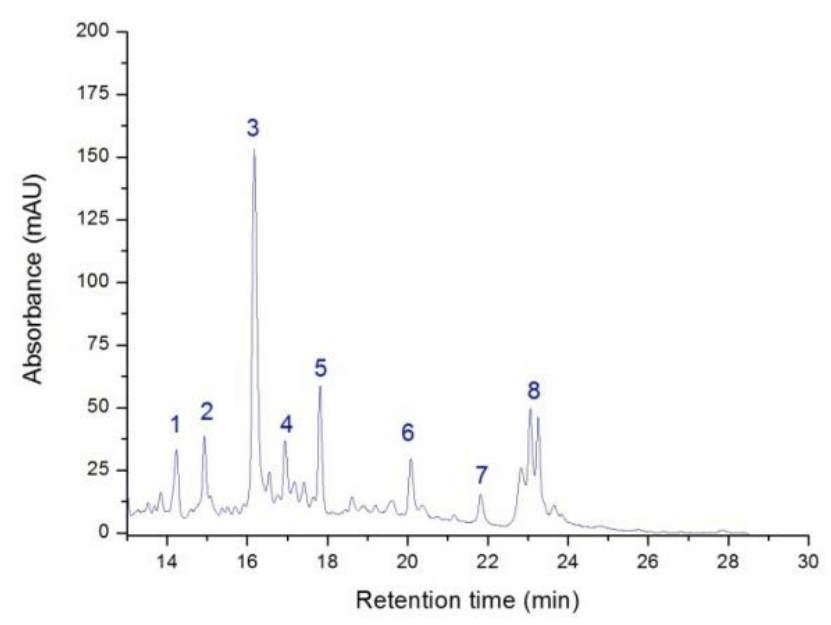

Figure 3. HPLC chromatogram of raspberry extracts

Raspberry. For raspberry extract total flavonol glycosides values were much lower than values obtained for total phenolic acids (Table 2). Hydroxybenzoic acids identified in analyzed extract were as follow: ellagic acidpentoside (peak 1), ellagic acid-pentoside (peak 2), ellagic acid (peak 3) and ellagic acid-acetyl-xyloside (peak 8) (Figure 3). The extract was found to have high amount of ellagic acid (30.63 mg/100 g FW) which is in agreement with a recent study published by Pimpão R.C., \& al., 2013 [29]. For flavonol glycosides, only quercetin in conjugated forms linked to sugars were detected: quercetingalactosyl-rhamnoside (peak 4), quercetin-glucosylrutinoside (peak 5), quercetin-galactoside (peak 6), and quercetin-glucoside (peak 7). Quercetin-glucosylrutinoside was found to more abundant $(9.85 \mathrm{mg} / 100 \mathrm{~g}$ FW) while quercetin-galactoside was found in the lower amount $(6.83 \mathrm{mg} / 100 \mathrm{~g} \mathrm{FW})$. The identification of other flavonoids such as kaempferol or (+)-catechin in previously literature data appears to vary based on analyzed berry genotype or varieties [2,31,32]. 
Blackberry. The contents of individual flavonols and total phenolic of blackberry extract obtained in this study are showed in Table 2. The identified compounds in order of the elution were: myricetin-galactoside, quercetinrutinoside (rutin), myricetin-arabinoside, quercetingalactoside, quercetin-glucoside (Figure 4). Myricetinarabinoside was found the predominant flavonol in blackberry extract, $36.92 \mathrm{mg} / 100 \mathrm{~g}$ FW. The obtained values (86.12 mg/100 g FW) are higher than ones reported by M.J. Cho \& al, 2005 [17] (15 mg/100 g FW), Siriwoharn \& Wrolstad, 2004 [33] (17.8, respectively 11.6 mg/100G FW. Higher values were reported by Henning \& al, 1981 [34].

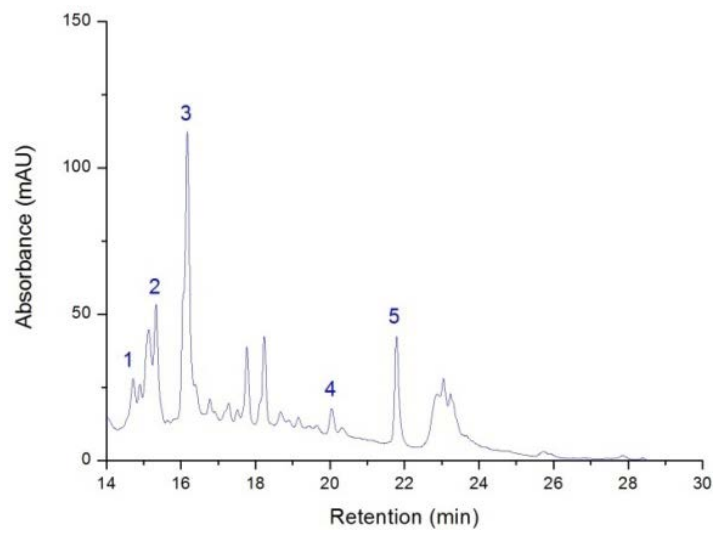

Figure 4. HPLC chromatogram of blackberry extracts
Cranberries. Data regarding elution order of flavonols glycosides on cranberries extract are showed in Figure 5. The identified compounds were: myricetin-galactoside, quercetin-rutinoside (rutin), myricetin-arabinoside, myricetin-glucoside, quercetin-galactoside, quercetinacetyl-glucoside, quercetin-glucoside, and quercetinrhamnoside. The predominant flavonol in this berries were quercetin-galactoside (12.02 mg/100 g FW) follow by quercetin-acetyl-glucoside (13.58 mg/100 g FW) and quercetin-glucoside (14.25 mg/100 g FW). The obtained results are comparable with the values reported by $\mathrm{G}$. Borges \& al. 2010 [25].

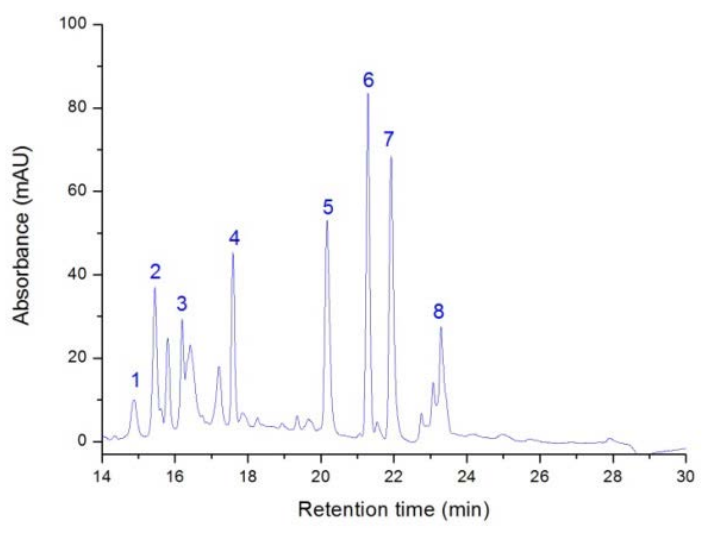

Figure 5. HPLC chromatogram of cranberry extracts

Table 2. HPLC-MS identification and quantification of phenolic acid and flavonol glycoside content in blueberry, blackberry, raspberries, and cranberries expressed as rutin and chlorogenic acid equivalents (mg/100 g FW of fruits)

\begin{tabular}{|c|c|c|c|c|c|}
\hline peak & $t_{R}(\min )$ & $\begin{array}{c}{[\mathrm{M}-\mathrm{H}]^{+}} \\
(\mathrm{m} / \mathrm{z})\end{array}$ & $\begin{array}{c}\text { UV } \lambda_{\max } \\
(\mathrm{nm})\end{array}$ & Phenolic compound & Concentration (mg rutin/chlorogenic acid eq. \pm sd/100 g FW) \\
\hline \multicolumn{6}{|c|}{ Blueberry } \\
\hline 1 & 12,4 & 355,181 & 278,320 & Chlorogenic Acid & $5.13 \pm 0.02$ \\
\hline 2 & 13,9 & 181,163 & 278,320 & Caffeic Acid & $7.5 \pm 0.23$ \\
\hline 3 & 15,4 & 611,303 & 270,360 & Quercetin-rutinoside (Rutin) & $65.96 \pm 1.28$ \\
\hline 4 & 20,2 & 465,303 & 265,355 & Quercetin-galactoside & $8.16 \pm 0.14$ \\
\hline 5 & 22,0 & 465,303 & 265,355 & Quercetin-glucoside & $7.33 \pm 0.14$ \\
\hline \multicolumn{6}{|c|}{ Rasberry } \\
\hline 1 & 14,2 & 435,303 & 254,360 & Ellagic acid-pentoside & $7.40 \pm 0.19$ \\
\hline 2 & 14,9 & 435,303 & 254,360 & Ellagic acid-pentoside & $8.61 \pm 0.05$ \\
\hline 3 & 16,0 & 303 & 262,363 & Ellagic acid & $30.63 \pm 0.03$ \\
\hline 4 & 16,9 & 611,303 & 270,360 & Quercetin-galactosyl-rhamnoside & $7.18 \pm 0.01$ \\
\hline 5 & 17,8 & $744,611,303$ & 270,360 & Quercetin-glucosyl-rutinoside & $9.85 \pm 0.06$ \\
\hline 6 & 21,2 & 465,303 & 265,355 & Quercetin-galactoside & $6.83 \pm 0.09$ \\
\hline 7 & 22,0 & 465,303 & 265,355 & Quercetin-glucoside & $5.73 \pm 0.59$ \\
\hline 8 & 23,0 & 477,303 & 255,360 & Ellagic acid-acetyl-xyloside & $8.94 \pm 0.12$ \\
\hline \multicolumn{6}{|c|}{ Blackberry } \\
\hline 1 & 14,7 & 481,319 & 272,358 & Myricetin-galactoside & $14.65 \pm 0.22$ \\
\hline 2 & 15,4 & 611,303 & 270,360 & Quercetin-rutinoside (Rutin) & $12.00 \pm 0.10$ \\
\hline 3 & 16,2 & 451,319 & 270,358 & Myricetin-arabinoside & $36.92 \pm 0.06$ \\
\hline 4 & 20,2 & 465,303 & 265,355 & Quercetin-galactoside & $13.46 \pm 0.05$ \\
\hline 5 & 22,0 & 465,303 & 265,355 & Quercetin-glucoside & $9.09 \pm 0.03$ \\
\hline \multicolumn{6}{|c|}{ Cranberry } \\
\hline 1 & 14,7 & 481,319 & 272,358 & Myricetin-galactoside & $4.61 \pm 0.09$ \\
\hline 2 & 15,4 & 611,303 & 270,360 & Quercetin-rutinoside (Rutin) & $8.01 \pm 0.11$ \\
\hline 3 & 16,2 & 451,319 & 270,358 & Myricetin-arabinoside & $5.68 \pm 0.06$ \\
\hline 4 & 17,5 & 481,319 & 272,358 & Myricetin-glucoside & $8.68 \pm 0.09$ \\
\hline 5 & 20,2 & 465,303 & 265,355 & Quercetin-galactoside & $12.02 \pm 0.04$ \\
\hline 6 & 21,2 & 507,303 & 268,357 & Quercetin-acetyl-glucoside & $13.58 \pm 0.09$ \\
\hline 7 & 22,0 & 465,303 & 265,355 & Quercetin-glucoside & $14.25 \pm 0.02$ \\
\hline 8 & 23,2 & 449,303 & 267,356 & Quercetin-rhamnoside & $7.32 \pm 0.03$ \\
\hline
\end{tabular}

The data expressed as Mean \pm SD of $\mathrm{mg} / 100 \mathrm{~g}$ FW of fruits.

\section{Conclusions}

The berries analyzed in this study contain a large amount of phenolic compounds and also exhibit high antioxidant activity. HPLC-ESI/MS analysis was successfully used for phenolic acid and flavonol glycosides identification and quantification. Following chromatographic separation 16 compounds were identified in analyzed berries. Considerable variation regarding 
antioxidant activity of obtained extracts was found, the higher antioxidant activity was obtained by blueberry extract while the lower value was obtained for cranberries extract. The identified flavonols in the analyzed berries were mainly represented by quercetin derivates as galactoside, rutinoside and also myricetin-galactoside were the major flavonol quantified. The obtained results provide valuable information regarding the potential health benefits of different berries due to their antioxidant activity and high amount of bioactive compounds content.

\section{Acknowledgement}

This paper was published under the frame of European Social Fund, Human Resources Development Operational Programme 2007-2013, project no. POSDRU/159/1.5/S/132765

\section{References}

[1] Yun, J.M., et al., Delphinidin, an anthocyanidin in pigmented fruits and vegetables, induces apoptosis and cell cycle arrest in human colon cancer HCT116 cells. Mol Carcinog, 2009. 48 (3): p. 260-70.

[2] Maatta-Riihinen, K.R., A. Kamal-Eldin, and A.R. Torronen, Identification and quantification of phenolic compounds in berries of Fragaria and Rubus species (family Rosaceae). J Agric Food Chem, 2004. 52 (20): p. 6178-87.

[3] Kao, E.S., et al., Anti-inflammatory potential of flavonoid contents from dried fruit of Crataegus pinnatifida in vitro and in vivo. J Agric Food Chem, 2005. 53 (2): p. 430-6.

[4] Hertog, M.G.L., P.C.H. Hollman, and B. van de Putte, Content of potentially anticarcinogenic flavonoids of tea infusions, wines, and fruit juices. Journal of Agricultural and Food Chemistry, 1993. 41 (8): p. 1242-1246.

[5] Jing, P. and M.M. Giusti, Contribution of Berry Anthocyanins to Their Chemopreventive Properties, in Berries and Cancer Prevention, N.P. Seeram and G.D. Stoner, Editors. 2011, Springer New York. p. 3-40.

[6] Routray, W. and V. Orsat, Blueberries and Their Anthocyanins: Factors Affecting Biosynthesis and Properties. Comprehensive Reviews in Food Science and Food Safety, 2011. 10 (6): p. 303320.

[7] Wang, J. and G. Mazza, Inhibitory effects of anthocyanins and other phenolic compounds on nitric oxide production in LPS/IFNgamma-activated RAW 264.7 macrophages. J Agric Food Chem, 2002. 50 (4): p. 850-7.

[8] de Pascual-Teresa, S. and M. Sanchez-Ballesta, Anthocyanins: from plant to health. Phytochemistry Reviews, 2008.7 (2): p. 281299.

[9] Wang, L.S. and G.D. Stoner, Anthocyanins and their role in cancer prevention. Cancer Lett, 2008. 269 (2): p. 281-90.

[10] Al-Hajj, N.Q.M., et al., Chemical Composition and Antioxidant Activity of the Essential Oil of Pulicaria Inuloides. Journal of Food and Nutrition Research, 2014. 2 (5): p. 221-227.

[11] Lock, K., et al., The global burden of disease attributable to low consumption of fruit and vegetables: implications for the global strategy on diet. Bull World Health Organ, 2005.83 (2): p. 100-8.

[12] Crozier, A., et al., Secondary Metabolites in Fruits, Vegetables, Beverages and Other Plant-based Dietary Components, in Plant Secondary Metabolites 2007, Blackwell Publishing Ltd. p. 208302.

[13] Ahmadiani, N., et al., Anthocyanins contents, profiles, and color characteristics of red cabbage extracts from different cultivars and maturity stages. J Agric Food Chem, 2014. 62 (30): p. 7524-31.

[14] WANG H., C.G., and PRIOR R. L., , Oxygen Radical Absorbing Capacity of Anthocyanins. J Agric Food Chem, 1997. 45: p. 304309.
[15] Tamura, H. and A. Yamagami, Antioxidative activity of monoacylated anthocyanins isolated from Muscat Bailey A grape. Journal of Agricultural and Food Chemistry, 1994. 42 (8): p. 1612-1615.

[16] Haleem, M.A., et al., Increasing antioxidant intake from fruits and vegetables: practical strategies for the Scottish population. J Hum Nutr Diet, 2008. 21 (6): p. 539-46.

[17] Cho, M.J., et al., Flavonoid glycosides and antioxidant capacity of various blackberry, blueberry and red grape genotypes determined by high-performance liquid chromatography/mass spectrometry. Journal of the Science of Food and Agriculture, 2004. 84 (13): p. 1771-1782.

[18] Zheng, W. and S.Y. Wang, Oxygen radical absorbing capacity of phenolics in blueberries, cranberries, chokeberries, and lingonberries. J Agric Food Chem, 2003.51 (2): p. 502-9.

[19] Shahidi, F. and P.K. Wanasundara, Phenolic antioxidants. Crit Rev Food Sci Nutr, 1992. 32 (1): p. 67-103.

[20] Mandal, S.M. and S. Dey, LC-MALDI-TOF MS-based rapid identification of phenolic acids. J Biomol Tech, 2008. 19 (2): p. 116-21.

[21] Kajdžanoska, M., V. Gjamovski, and M. Stefova, HPLC-DADESI-MSn identification of phenolic compounds in cultivated strawberries from Macedonia. 2010. Vol. 29. 2010.

[22] Hingse, S., S. Digole, and U. Annapure, Method development for simultaneous detection of ferulic acid and vanillin using highperformance thin layer chromatography. Journal of Analytical Science and Technology, 2014. 5 (1): p. 1-9.

[23] Hong, Y.S., et al., Metabolomics reveals simultaneous influences of plant defence system and fungal growth in Botrytis cinereainfected Vitis vinifera cv. Chardonnay berries. J Exp Bot, 2012. 63 (16): p. 5773-85.

[24] Benzie, I.F. and J.J. Strain, Ferric reducing/antioxidant power assay: direct measure of total antioxidant activity of biological fluids and modified version for simultaneous measurement of total antioxidant power and ascorbic acid concentration. Methods Enzymol, 1999. 299: p. 15-27.

[25] Borges, G., et al., Identification of Flavonoid and Phenolic Antioxidants in Black Currants, Blueberries, Raspberries, Red Currants, and Cranberriest. Journal of Agricultural and Food Chemistry, 2009. 58 (7): p. 3901-3909.

[26] Moyer, R.A., et al., Anthocyanins, phenolics, and antioxidant capacity in diverse small fruits: vaccinium, rubus, and ribes. J Agric Food Chem, 2002. 50 (3): p. 519-25.

[27] Koca, I. and B. Karadeniz, Antioxidant properties of blackberry and blueberry fruits grown in the Black Sea Region of Turkey. Scientia Horticulturae, 2009. 121 (4): p. 447-450.

[28] Castrejón, A.D.R., et al., Phenolic profile and antioxidant activity of highbush blueberry (Vaccinium corymbosum L.) during fruit maturation and ripening. Food Chemistry, 2008. 109 (3): p. 564572.

[29] Pimpao, R.C., et al., Analysis of phenolic compounds in Portuguese wild and commercial berries after multienzyme hydrolysis. J Agric Food Chem, 2013. 61 (17): p. 4053-62.

[30] Kader, F., et al., Fractionation and identification of the phenolic compounds of Highbush blueberries (Vaccinium corymbosum, L.). Food Chemistry, 1996.55 (1): p. 35-40.

[31] Gavrilova, V., et al., Separation, characterization and quantification of phenolic compounds in blueberries and red and black currants by HPLC-DAD-ESI-MSn. J Agric Food Chem, 2011. 59 (8): p. 4009-18.

[32] Zafrilla, P., F. Ferreres, and F.A. Tomas-Barberan, Effect of processing and storage on the antioxidant ellagic acid derivatives and flavonoids of red raspberry (Rubus idaeus) jams. J Agric Food Chem, 2001. 49 (8): p. 3651-5.

[33] Iriwoharn, T.S. and R.E. Wrolstad, Polyphenolic Composition of Marion and Evergreen Blackberries. J Food Sci, 2004. 69 (4): p. FCT233-FCT240.

[34] Henning, W., Phenolics of fruit. XIV. Flavonol glycosides of strawberries (Fragaria $\times$ ananassa Duch), raspberries (Rubus idaeus L) and blackberries (Rubus fruticosus L) Z Lebensm Untersuch Forsch, 1981. 173: p. 180-187. 